\title{
DISEÑO E IMPLEMENTACIÓN DE UNA PLATAFORMA ROBÓTICA MÓVIL PARA IDENTIFICACIÓN DE MINAS TERRESTRES ANTIPERSONA EN DIFERENTES TERRENOS DEL TERRITORIO COLOMBIANO
}

\section{DESIGN AND IMPLEMENTATION OF A MOBILE ROBOT PLATFORM FOR IDENTIFICATION OF LAND MINES IN VARIOUS FIELDS OF COLOMBIA}

\author{
Sanabria Bustos J.R ${ }^{1}$, Rentería Aguas J.K ${ }^{2}$, Casas Díaz C.A ${ }^{3}$, R oa-Guerrero E.E ${ }^{4}$
}

RESUMEN

\begin{abstract}
Actualmente la labor de desminado realizada por la fuerza pública es considerada un trabajo con un alto índice de mortalidad. El propósito del artículo fue desarrollar e implementar una plataforma robótica teledirigida para la identificación de minas terrestres antipersona en diferentes terrenos; partiendo de un sistema de evasión de obstáculos, mediante el algoritmo de VFH (Vector Field Histogram), mediante el software LabView Robotics. Los resultados obtenidos mostraron que el sistema de tracción tipo oruga permite un mejor desplazamiento, más agarre en los diferentes terrenos en comparación con el sistema de tracción tipo rueda, además se obtuvo un $87,5 \%$ de exactitud en la identificación de minas, a partir de un conjunto de 8 materiales diferentes, en comparación con el sistema MICRONTA 4003 utilizado actualmente. El sistema implementado contribuye a la detección de objetos peligrosos sobre diferentes terrenos, convirtiéndose en una alternativa para disminuir los indices de mortalidad en las tareas de desminado.
\end{abstract}

Palabras clave.- Maniobrabilidad, MICRONTA, Plataforma teledirigida, Sistema de tracción.

\begin{abstract}
In the present time the mine clearance by the security forces is considered a job with a high mortality rate. The purpose of this article was to develop and implement a robotic platform remote control for identification of antipersonnel landmines in various fields; starting from an obstacle avoidance system, for this propose we using the algorithm VFH (Vector Field Histogram) through the LabVIEW Robotics software. The results have showed that the caterpillar type traction system allows better movement, more grip in all fields compared to the type wheel drive system, plus $87.5 \%$ accuracy in identifying mines was obtained from a set of 8 different materials, compared with the currently used system MICRONTA 4003. The implemented system contributes to the detection of dangerous objects on different grounds, becoming an alternative to reduce mortality rates in demining.
\end{abstract}

Key words.- Maneuverability, MICRONTA, Remote control platform, Drive system.

La Revista Científica TECNIA protege los derechos de autor bajo la Licencia 4.0 de Creative Commons: Attribution 4.0 International (CC BY 4.0)

${ }^{1}$ Integrante del Grupo de Investigación GIGATT y estudiante de Ingeniería Electrónica en la Universidad de Cundinamarca (UDEC) Fusagasugá, Colombia, ${ }^{2}$ Integrante del Grupo de Investigación GIGATT y estudiante de Ingeniería Electrónica en la Universidad de Cundinamarca (UDEC) Fusagasugá, Colombia, ${ }^{3}$ Director del Grupo de Investigación en Generación Apropiación y Transferencia de Tecnologías (GIGATT), Fusagasugá, Colombia, ${ }^{4}$ Profesor en Ingeniería Electrónica en la Universidad de Cundinamarca (UDEC) Fusagasugá, Colombia, e integrante del Grupo en Tecnologías de la Información y las Comunicaciones. 


\section{INTRODUCCIÓN}

Hoy en día Colombia es uno de los países de América Latina donde aún se siembran artefactos explosivos [1]. Actualmente el Programa Presidencial para la Acción Integral contra Minas Antipersonal de la Republica de Colombia [2] indica que desde el año 1990 hasta junio del 2013 las minas antipersonal han cobrado cerca de 10.445 víctimas, convirtiéndose así en una problemática social y económica; para disminuir el número de víctimas de este tipo de artefactos se han utilizado diversos métodos para la detección, como el uso de perros entrenados, la detección por parte del técnico experto dependiendo de la topografía del terreno, así como también el uso de plataformas robóticas móviles para facilitar el trabajo realizado por el ser humano en tareas repetitivas o que representan peligro para su integridad.

Estos métodos representan un riesgo potencial para los técnicos expertos que deben estar en el lugar exacto donde se encuentra la mina antipersona para operar, en Colombia y muchos otros países a pesar del riego que representa esta actividad aún se sigue realizando de forma manual, además, hoy en día son utilizados diversos materiales artesanales improvisados por parte de los grupos armados para que no puedan ser detectadas de manera fácil.

Con el fin de disminuir el riesgo que representa para el operario de desminado, el propósito de este trabajo es desarrollar un prototipo de plataforma robótica móvil que logre desplazarse con facilidad dentro del área minada; y también pueda detectar un tipo de artefacto explosivo. Esta deberá ser flexible, programable y modular, lo cual permitiríasu masiva fabricación; convirtiéndose así en una herramienta útil para desminado de las Fuerzas militares (FF.MM), logrando de forma directa la disminución de muertes humanas y así mismo el retorno de la población civil a sus parcelas y retomar así sus actividades de agricultura y ganadería contribuyendo en la solución de esta gran problemática social.
Actualmente, se han desarrollado un sin número de proyectos para detección de minas antipersona desde detectores de metales a partir de campos magnéticos [3] hasta el desarrollo de plataformas robóticas F-Track desarrolladas por [4] las cuales pueden desplazarse hacia adelante y hacia atrás y acondicionado con 8 servo motores logrando un amplio abanico de movimientos.

\section{MATERIALES Y MÉTODOS}

La metodología implementada en la plataforma robótica experimental está compuesta de tres fases. La Fig. 1, describe la metodología mediante una representación de los módulos que componen la herramienta a través de un diagrama de flujo.

A continuación se describen cada uno de los módulos de la metodología:

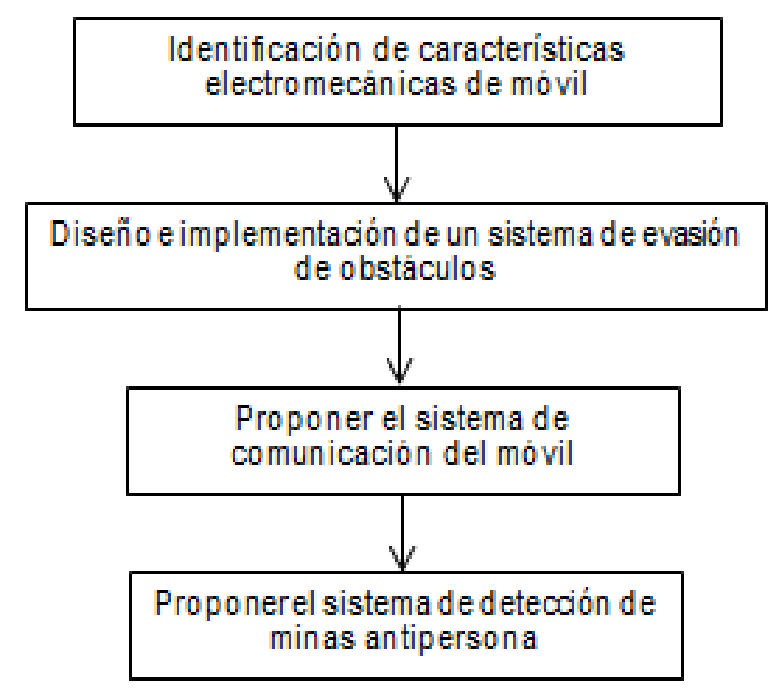

Fig.1 Metodología implementada en la plataforma robótica móvil.

\section{Fase I}

Identificar las características electromecánicas de la plataforma robótica móvil basándose en diferentes terrenos de aplicación. 
A. Definir los criterios de selección para implementar el tipo de tracción del móvil.

B. Obtener las especificaciones técnicas con respecto al tamaño y capacidad (carga, consumo energético) del sistema móvil.

C. Determinar el sistema de alimentación que cumpla con las exigencias del trabajo establecido para la propuesta de móvil.

D. Realización de pruebas de desempeño en los terrenos establecidos.

\section{Fase II}

Diseñar e implementar el sistema de detección de obstáculos para el desplazamiento de la plataforma robótica en diferentes terrenos.

A. Seleccionar los sensores adecuados para el desplazamiento de la plataforma robótica a través de obstáculos.

B. Evaluar el desempeño de la plataforma robótica móvil sobre diferentes tipos de terrenos y obstáculos.

\section{Fase III}

Proponer el sistema de comunicación del móvil.

A. Identificar estrategias de comunicación que permitan establecer una interfaz para comunicación con la plataforma robótica.
B. Validar la maniobrabilidad del sistema robótico mediante el sistema de comunicación.

\section{Fase IV}

Proponer el sistema de detección de minas antipersona

A. Seleccionar los sensores adecuados para la identificación de los artefactos explosivos.

B. Validar la detección de los materiales hallados con el sistema MICRONTA de uso privativo de las fuerzas militares [5].

\section{RESULTADOS}

El desarrollo de esta herramienta requiere de la combinación de diversos conocimientos como la mecánica, electrónica, la informática y la física en especial la electrónica que aportará el desarrollo de un sistema de comunicación que de operatividad hombre - máquina para el desplazamiento en diferentes terrenos.

Fase I.- La primera fase probada fue identificar de las características electromecánicas de la plataforma robótica móvil tipo oruga y rueda. Ver Tabla 1. 
Tabla 1. Especificaciones del tipo de tracción del móvil.

\section{ROBOT TIPO RUEDA}

ROBOT TIPO ORUGA

Son la solución más simple y eficiente de Sustituye las ruedas por mecanismo movilidad en terrenos suficientemente duros oruga para aumentar la tracción.

Como posee una rueda loca, la dirección seLa dirección se consigue parando una de consigue parando una de las ruedas olas Orugas o haciéndolas girar en sentido haciéndola girar en sentido contrario. contrario.

Tipo de desplazamiento veloz y económico Limitaciones en velocidad y economía

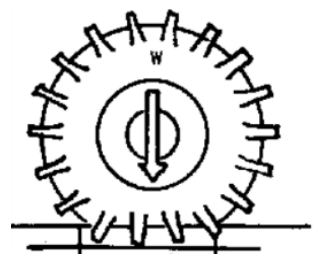

La definición de las especificaciones técnicas con respecto al tamaño y capacidad (carga, consumo energético) del sistema móvil. De acuerdo a los

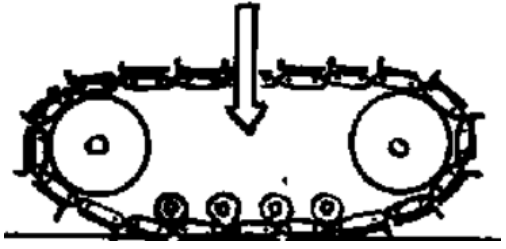

sistemas planteados se construyen dos plataformas de desplazamiento con las siguientes características mostradas en la Tabla 2:

Tabla 2. Criterios de selección del tipo de tracción del móvil.

\section{ESPECIFICACIONES TECNICAS DE LAS PLATAFORMAS ROBOTICAS}

\begin{tabular}{ccc}
\hline Dimensiones & Plataforma tipo oruga & Plataforma tipo (rueda) \\
Ancho & $39 \mathrm{~cm}$ & $39 \mathrm{~cm}$ \\
Largo & $37 \mathrm{~cm}$ & $41 \mathrm{~cm}$ \\
Alto & $24 \mathrm{~cm}$ & $16 \mathrm{~cm}$ \\
Batería & Se requieren $12 \mathrm{v}$ a $3 \mathrm{~A} / \mathrm{h}$ & Se requieren $12 \mathrm{v} \mathrm{a} 3 \mathrm{~A} / \mathrm{h}$ \\
\hline
\end{tabular}

Posteriormente se diseñó la plataforma robótica móvil en 3D mediante el software Creo Elemens de Pitsco teniendo en cuenta las especificaciones de diseño para el sistema de tracción tipo oruga y se realizaron modificaciones sobre el sistema de tracción tipo rueda que contábamos con la base de modelos 3D de pitsco, los resultados de los sistemas de tracción se muestran en la Fig. 2 y la Fig. 3 respectivamente. 


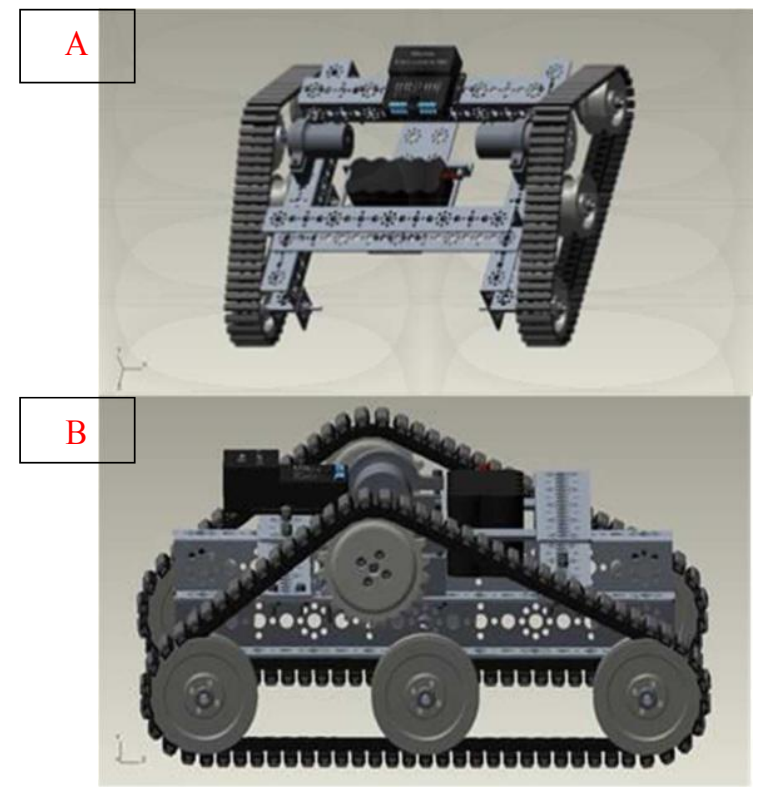

Fig. 2 Diseño de la plataforma robótica móvil tipo oruga en $3 D$ mediante el software Creo Elemens de Pitsco. A. Vista frontal, $B$. Vista laterales.

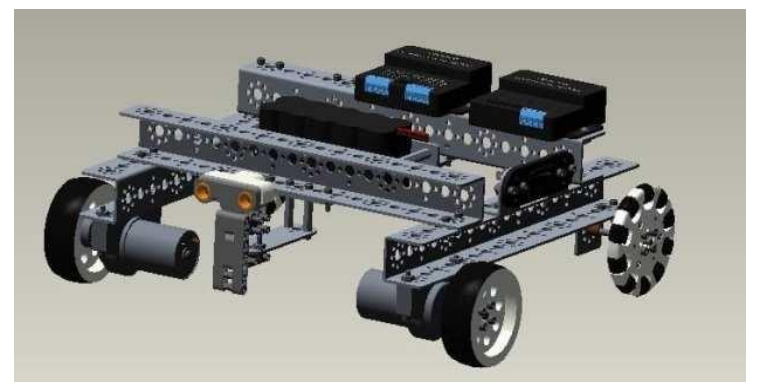

Fig. 3 Diseño de la plataforma robótica móvil tipo rueda en $3 D$ mediante el software Creo Elemens de Pitsco.

En la Tabla 2 se muestran las especificaciones del tipo de tracción del móvil tipo rueda y oruga, así mismo en la Tabla 3 se evidencian los resultados del tipo de tracción en diferentes terrenos de aplicación donde fueron probados, indicando que el tipo de tracción tipo oruga presenta mayores ventajas en terreno arenoso y césped, mientras que el tipo rueda presenta mayor velocidad en terreno liso y duro.

Tabla 3. Resultados del tipo de tracción del móvil.

\begin{tabular}{|c|c|c|c|}
\hline \multirow{2}{*}{ Prueba } & \multirow[t]{2}{*}{ Característica } & \multicolumn{2}{|c|}{ Tipo de Plataforma } \\
\hline & & Rueda & Oruga \\
\hline \multirow{4}{*}{$\begin{array}{l}\text { Desplazamiento } \\
\text { en terreno ilso o } \\
\text { duro }\end{array}$} & Velocidad & Buena & Regular \\
\hline & Maniobrabilidad & Buena & Buena \\
\hline & Tracción & Buena & Regular \\
\hline & Velocidad & Buena & Buena \\
\hline \multirow{3}{*}{$\begin{array}{l}\text { Desplazamiento } \\
\text { en cespeu } \\
\text { natural }\end{array}$} & Maniobrabilidad & Regular & Buena \\
\hline & Tracción & Regular & Buena \\
\hline & Velocidad & Regular & Buena \\
\hline \multirow{2}{*}{$\begin{array}{l}\text { Desplazamiento } \\
\text { en terreno } \\
\text { arenoso }\end{array}$} & Maniobrabilidad & Buena & Buena \\
\hline & Tracción & Regular & Buena \\
\hline
\end{tabular}

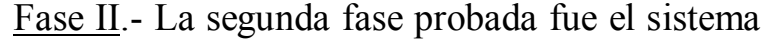
de detección de obstáculos para el desplazamiento de la plataforma robótica en diferentes terrenos. En esta fase se aplica el Software de National Instruments "LabView" con el fin de diseñar estrategias para la locomoción de plataformas robóticas (LabView Robotics), en ésta se configuran de forma práctica los sensores conectados a la plataforma de desarrollo SbRio. Así mismo, actuadores, algoritmos básicos para operación inteligente, percepción robusta y funciones de movimiento 
para mover el robot. Inicialmente se realizó la configuración adecuada para la comunicación entre el software LabView robotics y el robot ya que permite la programación por medio de la interfaz de Ethernet, posteriormente se realizó la configuración a través de la plataforma de desarrollo a trabajar la cual es la Starter Kit 2.0 sbRIO. Posteriormente, se determinó el tipo de sensor, los resultados de la investigación evidenciaron que los sensores ultrasónicos permiten la evasión de obstáculos, a continuación se muestra su implementación en LabView y los resultados del sensor ultrasónico en la Fig. 4 y Fig. 5 respectivamente.

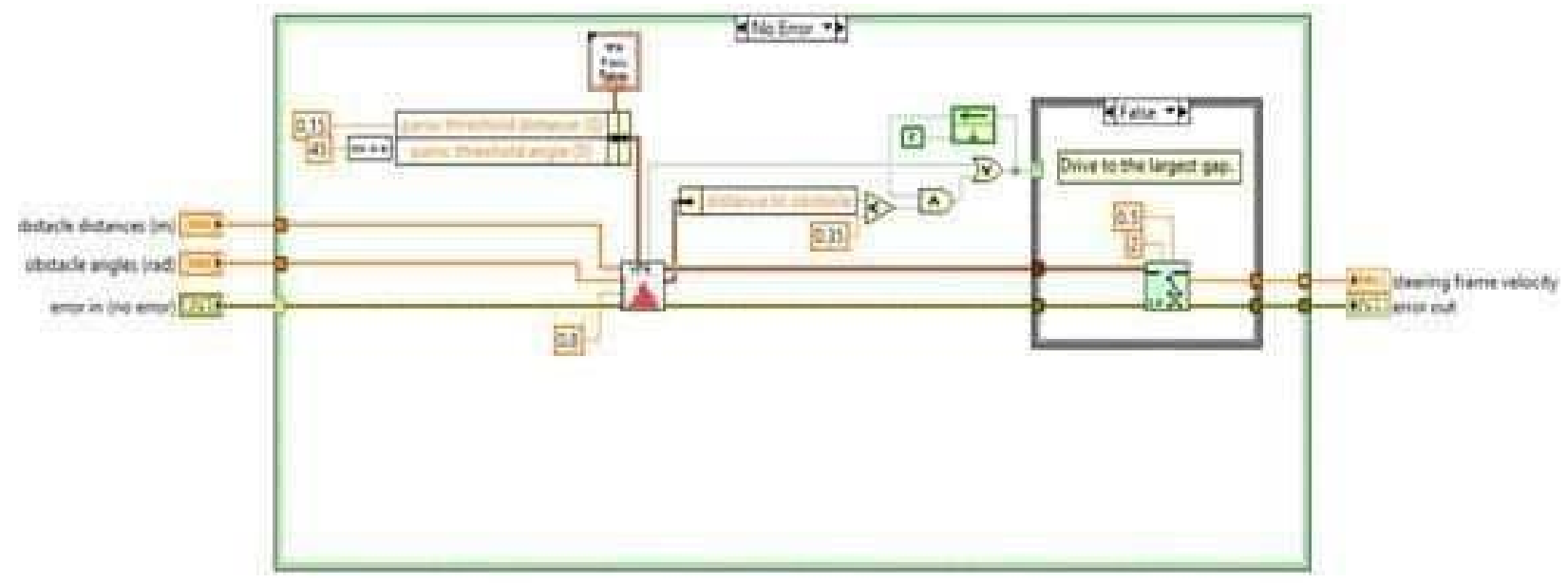

Fig. 4 Esquema en LabView del Histograma de campo vectorial, LabView Robotics.
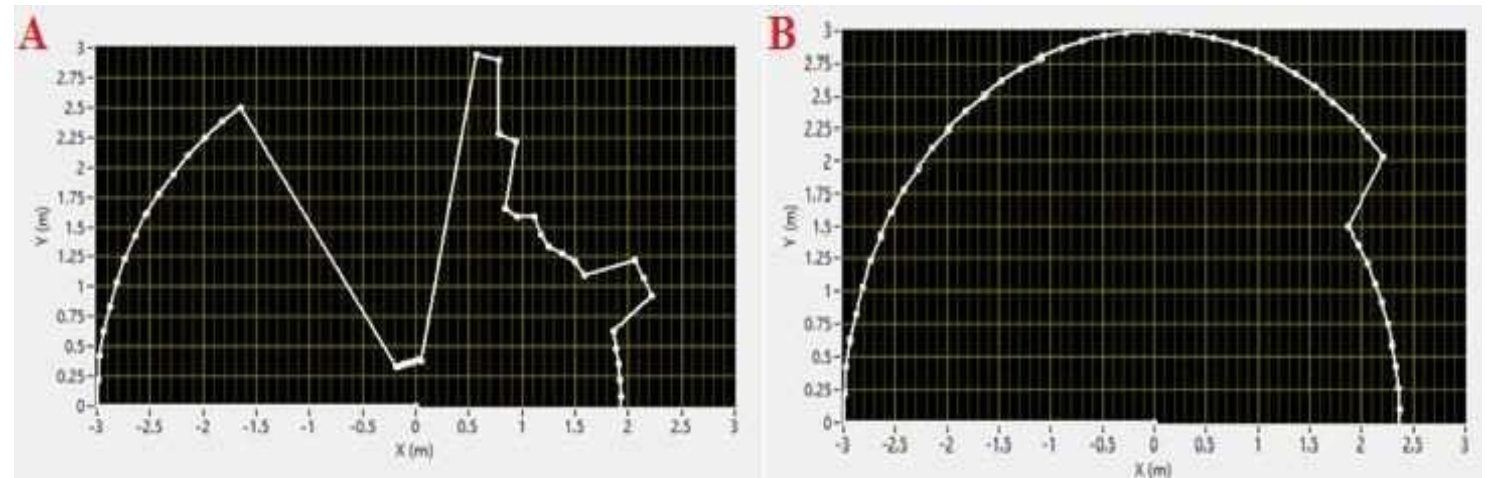

Fig. 5 A. Resultado del histograma de campo al tener objetos frente a él.

$B$. Histograma de campo sin tener un objeto frente a él.

A continuación se muestran las pruebas de evasión de obstáculos de los robots en la Fig. 6.



Fig. 6 Robot tipo oruga evadiendo obstáculos trayectoria (ABCD), Robot tipo rueda evadiendo obstáculos trayectoria $(E F G H)$. 
Fase III.- En la tercera fase se propuso el sistema de comunicación del móvil de acuerdo a los lineamientos requeridos se espera obtener un sistema de comunicación inalámbrico entre la plataforma robótica móvil (NI LabView Robotics DaNI) y el operario, que permita teledirigir el sistema en terrenos de difícil acceso, mediante la trasmisión de video en tiempo real de la plataforma robótica a través de una red inalámbrica bajo la supervisión de dicho operario, como se muestra en la Fig. 7.

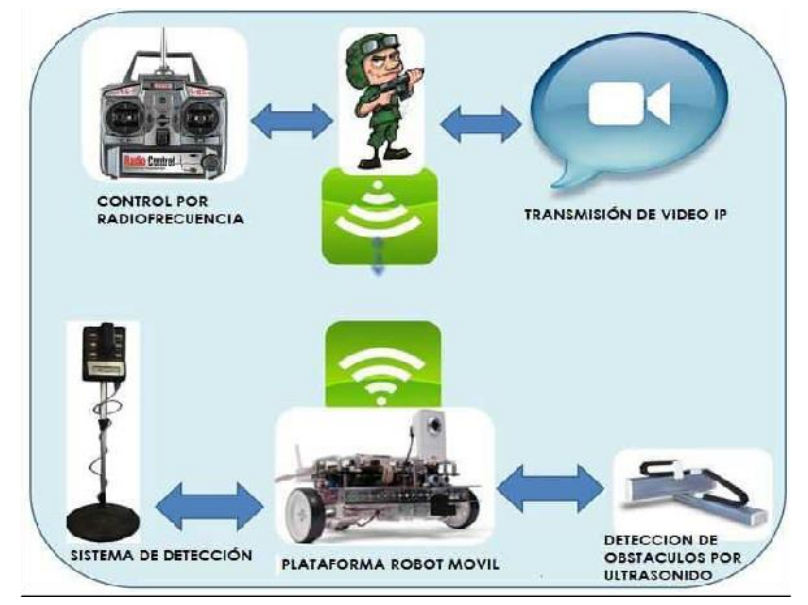

Fig. 7 Sistema de comunicación de la plataforma robótica móvil.
A continuación se muestran las pruebas de desempeño en la maniobrabilidad del sistema robótico móvil para diferentes terrenos en la Fig. 8.

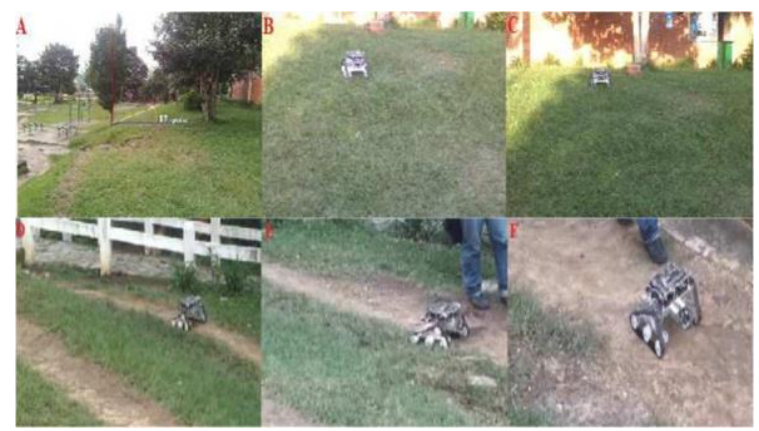

Fase IV.- Finalmente, en la cuarta fase se propuso el sistema para detectar artefactos de diferentes tipos de materiales que se consideran son utilizados para tal fin, fue utilizado un sensor capacitivo CM12-3004NA [5 y 7], el cual capta los materiales por medio de una frecuencia diferente, como se muestra en la Fig. 9.

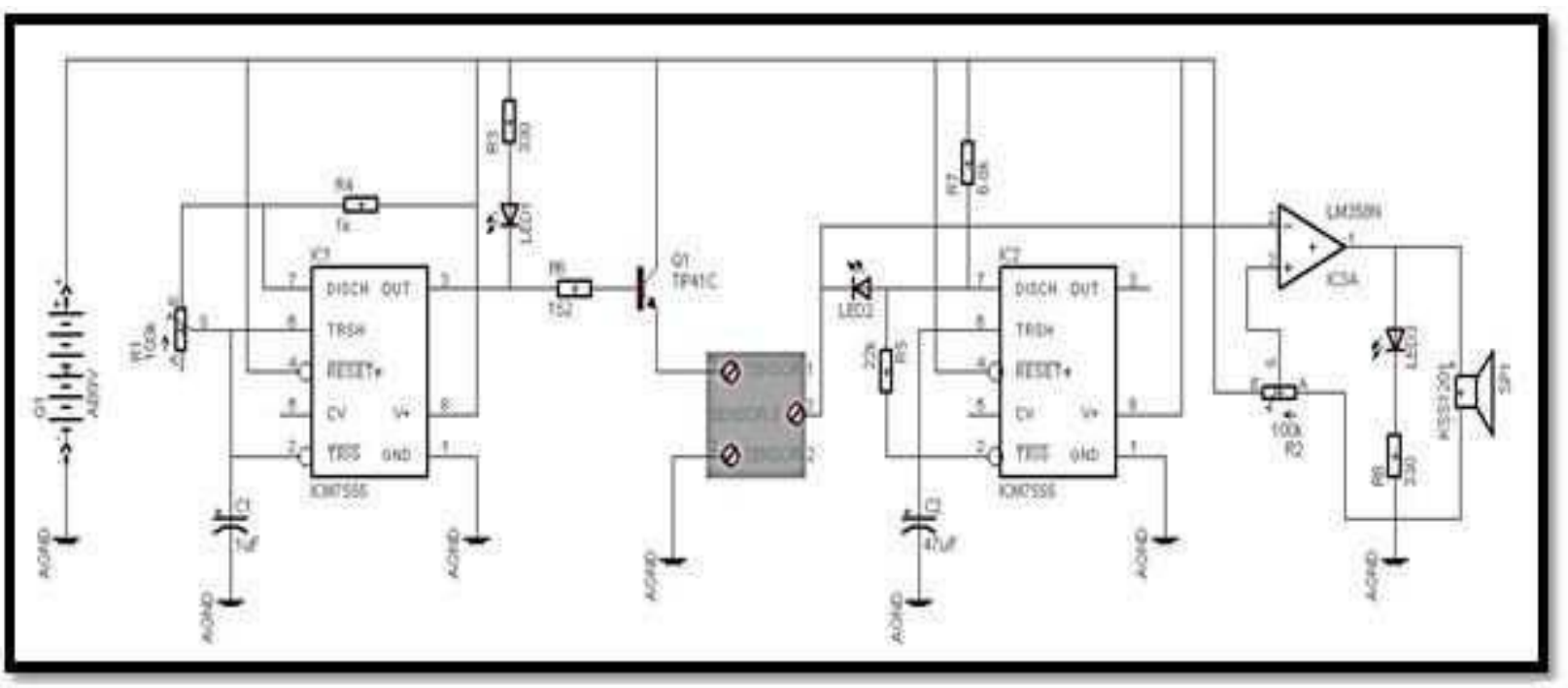

Fig. 9 Diagrama esquemático del circuito detector de metales.

A continuación en la Tabla 4, se muestran los resultados de la detección de los diferentes materiales utilizados en el proyecto con su respectiva frecuencia y su comparación con el sistema MICRONTA 4003 de uso privativo de las fuerzas militares (FF.MM) [6]. Evidenciando que el sistema Micronta solo detecta si es un objeto metálico o no, mientras 
que el sistema propuesto encuentra una frecuencia para cada objeto.

Tabla 4. Resultados de la comparación del sistema implementado vs el sistema MICRONTA 4003.

\begin{tabular}{cccc}
\hline \# De figura & Material & $\begin{array}{c}\text { Sistema } \\
\text { MICRONTA }\end{array}$ & $\begin{array}{c}\text { Sistema } \\
\text { propuesto }\end{array}$ \\
\hline 1 & Tungsteno & $\checkmark$ & $81.16 \mathrm{~Hz}$ \\
2 & Llave Expansiva & $\checkmark$ & $84.17 \mathrm{~Hz}$ \\
3 & Pinza punta plana & $\checkmark$ & $68.11 \mathrm{~Hz}$ \\
4 & potenciómetro & $X$ & $83.05 \mathrm{~Hz}$ \\
5 & Aluminio & $\checkmark$ & $85.07 \mathrm{~Hz}$ \\
6 & Estaño & $\checkmark$ & $83.33 \mathrm{~Hz}$ \\
7 & Moneda \$200 & $\checkmark$ & $92.92 \mathrm{~Hz}$ \\
8 & Llaves de cerradura & $\checkmark$ & $62.50 \mathrm{~Hz}$ \\
\hline
\end{tabular}

A continuación se muestran las frecuencias encontradas mediante el sistema implementado para cada material utilizado en el proyecto, permitiendo encontrar este tipo de materiales utilizados para tal fin en un rango de frecuencias determinadas.

En la Figura 10 hasta la Figura 17 se muestran las frecuencias determinadas por el sistema propuesto para cada material.



Fig. 10 Detección de frecuencia mediante el sistema implementado para el Tungsteno.

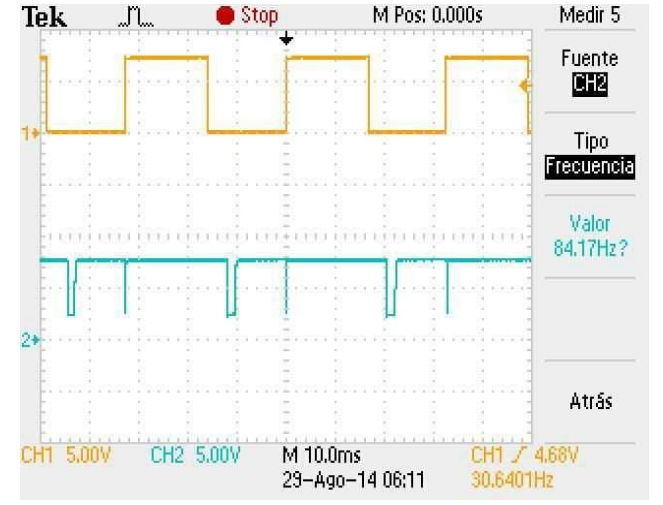

Fig. 11 Detección de frecuencia mediante el sistema implementado para la Llave expansiva.



Fig. 12 Detección de frecuencia mediante el sistema implementado para la pinza punta plana. 


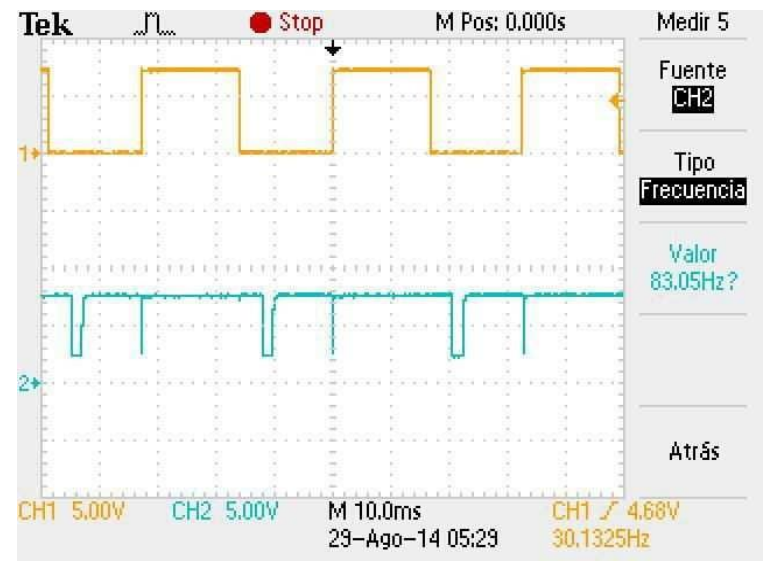

Fig. 13 Detección de frecuencia mediante el sistema implementado para el Potenciómetro.



Fig. 14 Detección de frecuencia mediante el sistema implementado para el Aluminio.

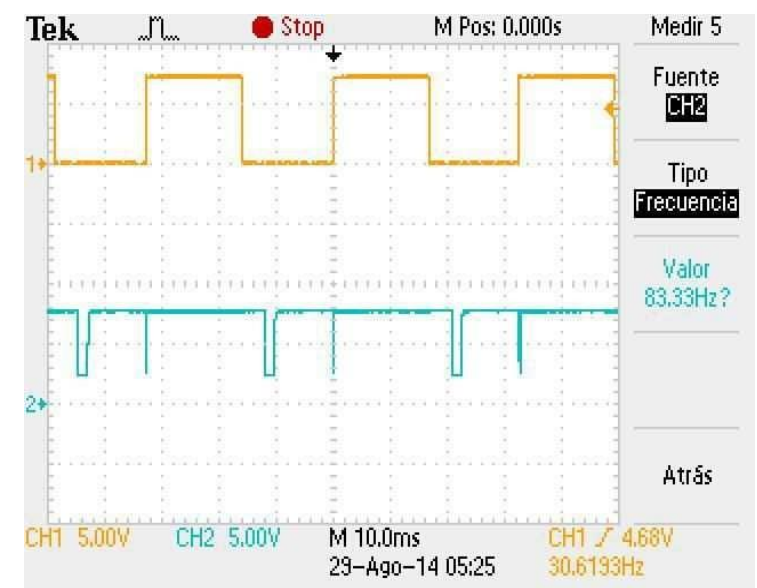

Fig. 15 Detección de frecuencia mediante el sistema implementado para el Estaño.

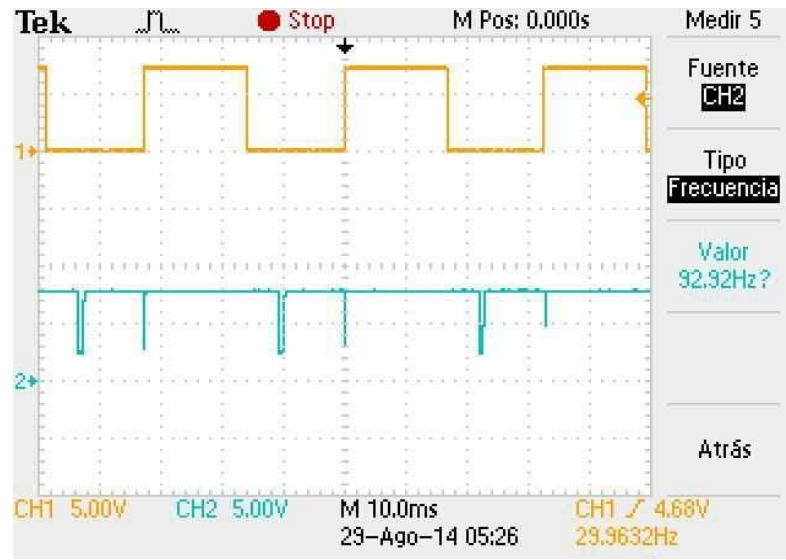

Fig. 16 Detección de frecuencia mediante el sistema implementado para la Moneda de 200 pesos.

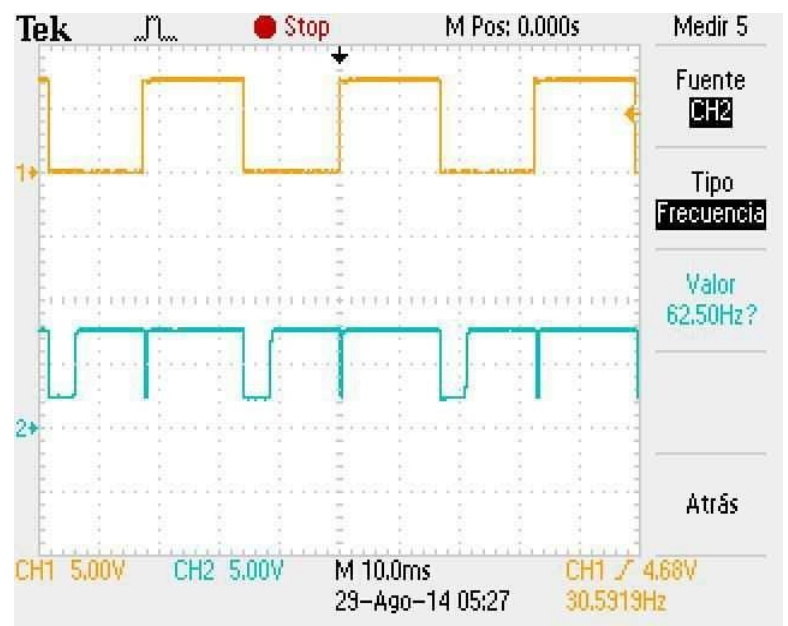

Fig. 17 Detección de frecuencia mediante el sistema implementado para las Llaves de cerradura.

\section{CONCLUSIONES}

En el presente artículo se muestra paso a paso el diseño e implementación de una plataforma robótica móvil basada en módulos de pitsco y software LabView, para la determinación de los diferentes objetos a partir de un sensor capacitivo CM12-3004NA. Los resultados del sistema mostrados en la tabla 3 han sido satisfactorios en cuanto a la detección de los materiales presentando una precisión de $87,5 \%$ en comparación con medidas obtenidas con el sistema MICRONTA 4003.

Se encontraron fallas en la detección de materiales en distancias mayores a $2 \mathrm{~cm}$ debido a 
la sintonización del sensor capacitivo. Debido a esto, el sistema deja de reconocer algunos materiales ocasionando errores en la identificación. Así mismo, se evidenció que una de las mayores ventajas es el desarrollo de una plataforma tipo oruga que pueda desplazarse en diferentes terrenos en comparación con la plataforma tipo rueda, por otra parte presentó ventajas en cuanto a velocidad, maniobrabilidad y tracción en diferentes terrenos como arenoso, liso y en césped.

Este sistema robótico móvil se convierte en una herramienta fundamental para la detección de minas antipersona en diferentes terrenos y el desarrollo de su metodología a partir del software LabView da una alternativa a nivel mundial, para reducción de los índices de mortalidad, pero futuras pruebas en diferentes terrenos y una mayor validación es necesaria para su uso militar.

Finalmente, se pretende desarrollar como trabajo futuro, sobre la plataforma implementada un sistema de detección de minas artesanales plásticas mediante el reconocimiento de imágenes, utilizando una cámara inalámbrica, con el fin de encontrar con más precisión las minas artesanales plásticas, las cuales no son fáciles de detectar mediante sensores metálicos.

\section{REFERENCIAS}

1. González, B., "Colombia y las Minas Antipersonal- Sembrando Minas Cosechando Muerte". Bogotá: Crear \& Comunicar, 2000.

2. http://www.accioncontraminas.gov.co. Programa Presidencial para Acción Integral Contra Minas Antipersona. Colombia, 2013.

3. Pino, J., “ Determinación de técnicas de detección de explosivos óptimas para el departamento de Antioquia. Medellín", Universidad Nacional de Colombia Facultad de Minas, Escuela de Procesos y Energía, 2009.

4. Marín M., "Control remoto de un robot imprimible de exteriores". Madrid

5. http://www.elmarkholding.eu/download/pro ducts/139/Cap acitive_Sensors, 2011.

6. http://www.whiteriverprep.com/vintage/radi oshack/rs.html. Radio Shack detectors de metals.

7. Pallas, R., "Sensores y acondicionadores de señal". Cuarta edición, editorial marcombo, 2003.

Correspondencia: msc.edgar.roa@hotmail.es

Recepción de originales: marzo 2015

Aceptación de originales: junio 2015 